\title{
Convenient Catalytic, Enantioselective Conjugate Reduction of Nitroalkenes Using $\mathrm{CuF}_{2}$
}

\author{
Constantin Czekelius and Erick M. Carreira* \\ Laboratorium für Organische Chemie, ETH Zürich \\ ETH Hönggerberg, HCI, H335, CH-8093 Zürich \\ carreira@org.chem.ethz.ch
}

\section{Experimental}

All reactions were carried out in dried glassware under an atmosphere of argon. Toluene was dried by passage over A-2 alumina (8 x 14 mesh, Macherey \& Nagel; activated at $300^{\circ} \mathrm{C}$ under nitrogen atmosphere for $12 \mathrm{~h}$ ).

Chemicals were purchased from Strem, Aldrich, Fluka, or Acros and used as is unless mentioned otherwise.

Evaporation of organic solutions was achieved by rotary evaporation with a water bath temperature below $40{ }^{\circ} \mathrm{C}$. Product purification by flash column chromatography was accomplished using silica gel 60 (32-63 $\mu \mathrm{m}$ particle size from Fluka or Brunschwig) at $0.1-0.3$ bar pressure. Technical grade solvents were used for chromatography and distilled prior to use. Thin-layer chromatography (TLC) was performed on Merck silica gel $60 \mathrm{~F}_{254}$ glass plates. Visualization was achieved by either fluorescence quenching or by staining with aqueous potassium permanganate solution.

Melting points were measured using a Büchi 510 melting point apparatus in open glass capillaries and are uncorrected. 
NMR spectra were recorded at room temperature on a Varian Mercury operating at $300 \mathrm{MHz}\left({ }^{1} \mathrm{H}\right)$ and $75 \mathrm{MHz}\left({ }^{13} \mathrm{C}\right)$ respectively. Residual solvent signals are internally referenced. Chemical shift $\delta$ is referred in terms of ppm, coupling constants are given in Hz. Following abbreviations classify the multiplicity: $\mathrm{s}=$ singlet, $\mathrm{d}=$ doublet, $\mathrm{t}=$ triplet, $\mathrm{q}=$ quartett, $\mathrm{m}=$ multiplet or unresolved, $\mathrm{br}=$ broad signal.

Infrared spectra were recorded on a Perkin-Elmer Spektrum RX I FT-IR System and reported in $\mathrm{cm}^{-1}$. Samples were prepared in thin film technique.

Combustion analysis was performed by the Mikroelementaranalytisches Laboratorium at ETH Zurich. Mass spectra were obtained from the MS Service of the ETH Zurich or the MS service of the University of Fribourg/Switzerland. MALDI-mass spectra were recorded using an Ion Spec Ultima HR FT-ICR MS MALDI-FT-ICR MS using the DHB-tl (2,5-dihydroxy-benzoic acid-two layers) method at 4.7 Tesla. High resolution EI mass spectra were performed on a Micromass AutoSpec Ultima and were calibrated with perfluorotributylamine (PFTBA) prior to data acquisition.

Enantiomeric ratios were determined with a Merck Hitachi LaChrom D-7000 HPLC using a Chiracel OD-H column, a Knauer differential refractometer, and hexane/isopropanol as eluents.

\section{General procedure for the reduction of nitroalkenes using copper(II)-fluoride}

In a $10 \mathrm{~mL}$ Schlenck-flask commercially available (Riedel-de-Haen), anhydrous copper(II)-fluoride $(5.1 \mathrm{mg}, 50 \mu \mathrm{mol})$ and $(R)-(S)-J O S I P H O S ~(33 \mathrm{mg}, 55 \mu \mathrm{mol})$ were dissolved in toluene $(5 \mathrm{~mL})$. After stirring for $60 \mathrm{~min}$ PMHS $(6.0 \mu \mathrm{L}, 0.10 \mathrm{mmol})$ was added followed by phenylsilane $(54 \mathrm{mg}, 62 \mu \mathrm{L}, 0.50 \mathrm{mmol})$ and water $(18.0 \mu \mathrm{L}, 1.00$ 
mmol). After stirring for $5 \mathrm{~min}$ the nitroolefin $(1.00 \mathrm{mmol})$ was added with vigorous stirring. After stirring for $12 \mathrm{~h}$, phenylsilane (108 mg, $124 \mu \mathrm{L}, 1.00 \mathrm{mmol}$ ) was added and stirring continued for $4 \mathrm{~h}$. TBAF solution ( $4 \mathrm{~mL}, 1.0 \mathrm{M}$ in THF, $4.0 \mathrm{mmol})$ was added and stirring was continued for $1 \mathrm{~h}$. Water $(20 \mathrm{~mL})$ was added and the mixture extracted with ether $(2 \times 30 \mathrm{~mL})$. After drying over sodium sulfate, the solvent was evaporated. Flash chromatography (hexane: EtOAc) provided the product as a colorless oil.

\section{General procedure for the reduction of nitroalkenes using copper(II)-fluoride and nitromethane}

In a $10 \mathrm{~mL}$ Schlenck-flask commercially available (Riedel-de-Haen), anhydrous copper(II)-fluoride $(5.1 \mathrm{mg}, 50 \mu \mathrm{mol})$ and $(R)-(S)-J O S I P H O S ~(33 \mathrm{mg}, 55 \mu \mathrm{mol})$ were dissolved in toluene $(5 \mathrm{~mL})$. After stirring for $60 \mathrm{~min}$ PMHS $(6.0 \mu \mathrm{L}, 0.10 \mathrm{mmol})$ was added followed by phenylsilane (32 mg, $37 \mu \mathrm{L}, 0.30 \mathrm{mmol})$ and water $(18.0 \mu \mathrm{L}, 1.00$ mmol). After stirring for $5 \mathrm{~min}$ nitromethane $(6.1 \mathrm{mg}, 5.4 \mu \mathrm{L}, 0.10 \mathrm{mmol})$ was added and stirring continued for $1 \mathrm{~h}$. Phenylsilane (108 mg, $124 \mu \mathrm{L}, 1.00 \mathrm{mmol})$ and the nitroolefin $(1.00 \mathrm{mmol})$ were added with vigorous stirring. After stirring for $12 \mathrm{~h}$, phenylsilane (54 $\mathrm{mg}, 62 \mu \mathrm{L}, 0.50 \mathrm{mmol})$ was added and stirring continued for $4 \mathrm{~h}$. TBAF solution $(4 \mathrm{~mL}$, $1.0 \mathrm{M}$ in THF, $4.0 \mathrm{mmol})$ was added and stirring was continued for $1 \mathrm{~h}$. Water $(20 \mathrm{~mL})$ was added and the mixture extracted with ether $(2 \times 30 \mathrm{~mL})$. After drying over sodium sulfate, the solvent was evaporated. Flash chromatography (hexane: EtOAc) provided the product as a colorless oil. 
<smiles>C/C(=C\[N+](=O)[O-])c1ccccc1</smiles>

(E)-2-Phenyl-1-nitro-1-propene:

${ }^{1} \mathbf{H}$ NMR (300 MHz, $\left.\mathbf{C D C l}_{3}, 25{ }^{\circ} \mathbf{C}\right): \delta=7.45(\mathrm{~m}, 5 \mathrm{H}$, arom. $H), 7.31(\mathrm{~m}, 1 \mathrm{H}, \mathrm{C} 1-H)$, $2.65(\mathrm{~m}, 3 \mathrm{H}, \mathrm{Me})$.

${ }^{13} \mathrm{C}$ NMR (75 MHz, $\left.\mathbf{C D C l}_{3}, 25{ }^{\circ} \mathbf{C}\right): \delta=149.8,138.1,136.1,130.2,128.9,126.7,18.6$. IR (film): $v=1623,1576,1514,1445,1375,1340,1257,921,835,765,738,696,609$ $\mathrm{cm}^{-1}$.

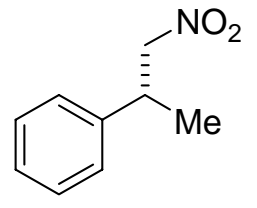

(R)-2-Phenyl-1-nitro-propane:

${ }^{1} \mathbf{H}$ NMR (300 MHz, $\left.\mathbf{C D C l}_{3}, 25{ }^{\circ} \mathbf{C}\right): \delta=7.34(\mathrm{~m}, 2 \mathrm{H}$, arom. $H$ ), 7.25 (m, 3H, arom. $H$ ), $4.58\left(\mathrm{dd}, J=12.1,7.5 \mathrm{~Hz}, 1 \mathrm{H}, \mathrm{C} 1-H^{1}\right), 4.49\left(\mathrm{dd}, J=12.1,8.1 \mathrm{~Hz}, 1 \mathrm{H}, \mathrm{C} 1-H^{2}\right), 3.64(\mathrm{~m}$, 1H, C2-H), 1.39 (d, $J=6.8 \mathrm{~Hz}, 3 \mathrm{H}, \mathrm{Me})$.

${ }^{13} \mathrm{C}$ NMR (75 MHz, $\left.\mathbf{C D C l}_{3}, 25{ }^{\circ} \mathbf{C}\right): \delta=140.7,128.8,127.4,126.8,81.8,38.7,18.8$.

IR (film): $v=3032,2974,2934,1604,1556,1496,1454,1432,1384,1332,1204,1211$, $1022,765,701 \mathrm{~cm}^{-1}$.

HPLC (Chiracel OD-H, hexane/iso-propanol 98:2, flow $0.9 \mathrm{ml} / \mathrm{min}$ ): $\mathrm{t}_{1}=7.58 \mathrm{~min}, \mathrm{t}_{2}=$ $9.03 \mathrm{~min}$. 
<smiles>C/C(=C\[N+](=O)[O-])c1ccc(Cl)cc1</smiles>

(E)-2-(4-Chloro-phenyl)-1-nitro-1-propene:

${ }^{1}$ H NMR (300 MHz, $\left.\mathbf{C D C l}_{3}, \mathbf{2 5}^{\circ} \mathbf{C}\right): \delta=7.40(\mathrm{~m}, 4 \mathrm{H}$, arom. $H$ ), $7.28(\mathrm{q}, J=1.6 \mathrm{~Hz}, 1 \mathrm{H}$, $\mathrm{C} 1-H), 2.62(\mathrm{~d}, J=1.6 \mathrm{~Hz}, 3 \mathrm{H}, \mathrm{Me})$.

${ }^{13}$ C NMR (75 MHz, [D 6 ]DMSO, $\left.25{ }^{\circ} \mathbf{C}\right): \delta=147.0,136.7,136.0,135.0,128.9,128.7$, 17.7.

IR (film): $v=3106,1622,1591,1515,1490,1435,1402,1374,1342,1255,1097,1012$, $921,823,756 \mathrm{~cm}^{-1}$.

Anal. Calcd for $\mathbf{C}_{9} \mathbf{H}_{8} \mathrm{NO}_{2} \mathrm{Cl}: \mathrm{C}, 54.70, \mathrm{H}, 4.08, \mathrm{~N}, 7.09$. Found: C, 54.83, H, 4.24, N, 6.91 .<smiles>C[C@H](C[N+](=O)[O-])c1ccc(Cl)cc1</smiles>

(R)-2-(4-Chloro-phenyl)-1-nitro-propane:

${ }^{1} \mathbf{H}$ NMR (300 MHz, $\left.\mathbf{C D C l}_{3}, 25{ }^{\circ} \mathbf{C}\right): \delta=7.31(\mathrm{~m}, 2 \mathrm{H}$, arom. $H), 7.16(\mathrm{~m}, 2 \mathrm{H}$, arom. $H)$, $4.52\left(\mathrm{dd}, J=15.8,7.8 \mathrm{~Hz}, 1 \mathrm{H}, \mathrm{C} 1-H^{1}\right), 4.48\left(\mathrm{dd}, J=15.8,7.8 \mathrm{~Hz}, 1 \mathrm{H}, \mathrm{C} 1-H^{2}\right), 3.62(\mathrm{~m}$, $1 \mathrm{H}, \mathrm{C} 2-\mathrm{H}), 1.36(\mathrm{~d}, J=7.2 \mathrm{~Hz}, 3 \mathrm{H}, \mathrm{Me})$.

${ }^{13} \mathrm{C}$ NMR (75 MHz, $\left.\mathbf{C D C l}_{3}, 25{ }^{\circ} \mathbf{C}\right): \delta=139.2,133.2,129.0,128.2,81.5,38.1,18.8$.

IR (film): $v=2974,1553,1495,1381,1094,1014,829,738 \mathrm{~cm}^{-1}$.

Anal. Calcd for $\mathrm{C}_{9} \mathrm{H}_{10} \mathrm{NO}_{2} \mathrm{Cl}$ : C, 54.15, H, 5.05, N, 7.02. Found: C, 54.35, H, 5.09, N, 6.85 .

HPLC (Chiracel OD-H, hexane/iso-propanol 98:2, flow $0.9 \mathrm{ml} / \mathrm{min}$ ): $\mathrm{t}_{1}=8.14 \mathrm{~min}, \mathrm{t}_{2}=$ $11.44 \mathrm{~min}$. 
<smiles>COc1ccc(/C(C)=C/[N+](=O)[O-])cc1</smiles>

(E)-2-(4-Methoxy-phenyl)-1-nitro-1-propene:

Mp: $31-32{ }^{\circ} \mathrm{C}$

${ }^{1}$ H NMR (300 MHz, $\left.\mathbf{C D C l}_{3}, 25{ }^{\circ} \mathbf{C}\right): \delta=7.43(\mathrm{~d}, J=8.1 \mathrm{~Hz}, 2 \mathrm{H}$, arom. H), $7.33(\mathrm{~s}, 1 \mathrm{H}$, C1-H), 6.94 (d, J=8.1 Hz, 2H, arom. H), 3.85 (s, 3H, OMe), 2.64 (s, 3H, Me).

${ }^{13} \mathrm{C}$ NMR (75 MHz, $\left.\mathbf{C D C l}_{3}, 25{ }^{\circ} \mathbf{C}\right): \delta=161.4,149.6,135.0,130.1,128.3,114.3,55.5$, 18.4 .

IR (film): $v=3107,2966,2937,2841,1602,1571,1513,1462,1374,1335,1295,1252$, $1184,1029,921,827,786 \mathrm{~cm}^{-1}$.

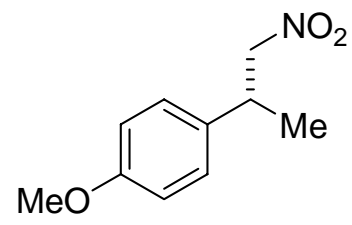

(R)-2-(4-Methoxy-phenyl)-1-nitro-propane:

${ }^{1}$ H NMR (300 MHz, $\left.\mathbf{C D C l}_{3}, 25{ }^{\circ} \mathbf{C}\right): \delta=7.15(\mathrm{~m}, 2 \mathrm{H}$, arom. $H), 6.87(\mathrm{~m}, 2 \mathrm{H}$, arom. $H)$, $4.51\left(\mathrm{dd}, J=11.8,7.5 \mathrm{~Hz}, 1 \mathrm{H}, \mathrm{C} 1-H^{1}\right), 4.45\left(\mathrm{dd}, J=11.8,8.1 \mathrm{~Hz}, 1 \mathrm{H}, \mathrm{C} 1-H^{2}\right), 3.79$ (s, 3H, OMe), 3.59 (m, 1H, C2-H), 1.36 (d, 3H, $J=7.2 \mathrm{~Hz}, \mathrm{Me})$.

${ }^{13} \mathbf{C}$ NMR (75 MHz, $\left.\mathbf{C D C l}_{3}, 25{ }^{\circ} \mathbf{C}\right): \delta=158.7,132.7,127.8,114.2,82.1,55.3,38.0$, 18.9 .

IR (film): $v=2968,1613,1551,1515,1459,1382,1300,1250,1181,1123,1025,832$, $772 \mathrm{~cm}^{-1}$.

HPLC (Chiracel OD-H, hexane/iso-propanol 98:2, flow $0.9 \mathrm{ml} / \mathrm{min}$ ): $\mathrm{t}_{1}=9.73 \mathrm{~min}, \mathrm{t}_{2}=$ $18.36 \mathrm{~min}$. 


\section{(E)-2-(Furan-2-yl)-1-nitro-1-propene:}

${ }^{1} \mathbf{H}$ NMR (300 MHz, $\left.\mathbf{C D C l}_{3}, 25{ }^{\circ} \mathbf{C}\right): \delta=7.67(\mathrm{~m}, 1 \mathrm{H}, \mathrm{C} 1-H), 7.54(\mathrm{~m}, 1 \mathrm{H}$, arom. $H)$, $6.90(\mathrm{~d}, J=3.7 \mathrm{~Hz}, 1 \mathrm{H}$, arom. $H), 6.55$ (m, 1H, arom. $H), 2.55$ (m, 3H, Me).

${ }^{13} \mathrm{C}$ NMR (75 MHz, $\left.\mathbf{C D C l}_{3}, 25{ }^{\circ} \mathbf{C}\right): \delta=150.5,145.5,136.6,132.9,115.8,112.8,14.9$.

IR (film): $v=3117,1610,1563,1504,1393,1343,1324,1266,1226,1161,1030,946$, $884,821,755,690 \mathrm{~cm}^{-1}$.

Anal. Calcd for $\mathbf{C}_{7} \mathbf{H}_{7} \mathrm{NO}_{3}$ : C, 54.90; H, 4.61; N, 9.15 Found: C, 54.91; H, 4.77; N, 8.99.

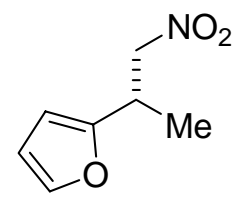

(R)-2-(Furan-2-yl)-1-nitro-propane:

${ }^{1} \mathbf{H}$ NMR (300 MHz, $\left.\mathbf{C D C l}_{3}, 25^{\circ} \mathbf{C}\right): \delta=7.35(\mathrm{dd}, J=1.9,0.6 \mathrm{~Hz}, 1 \mathrm{H}$, arom. $H), 6.31$ (dd, $J=3.4,1.9 \mathrm{~Hz}, 1 \mathrm{H}$, arom. $H$ ), 6.13 (m, 1H, arom. $H$ ), 4.67 (dd, $J=12.1,6.5 \mathrm{~Hz}, 1 \mathrm{H}$, C1- $\left.H^{1}\right), 4.43\left(\mathrm{dd}, J=12.1,8.1 \mathrm{~Hz}, 1 \mathrm{H}, \mathrm{C} 1-H^{2}\right), 3.74(\mathrm{~m}, 1 \mathrm{H}, \mathrm{C} 2-H), 1.38(\mathrm{~d}, J=7.2 \mathrm{~Hz}$, $3 \mathrm{H}, \mathrm{Me})$.

${ }^{13} \mathrm{C}$ NMR (75 MHz, $\left.\mathbf{C D C l}_{3}, 25{ }^{\circ} \mathbf{C}\right): \delta=153.7,141.9,110.2,105.9,79.5,32.5,16.2$.

IR (film): $v=2982,1555,1508,1433,1378,1150,1013,739 \mathrm{~cm}^{-1}$.

Anal. Calcd for $\mathrm{C}_{7} \mathrm{H}_{9} \mathrm{NO}_{3}: \mathrm{C}, 54.19 ; \mathrm{H}, 5.85 ; \mathrm{N}, 9.03$. Found: $\mathrm{C}, 54.30 ; \mathrm{H}, 6.12 ; \mathrm{N}$, 8.77 .

HPLC (two serial Chiracel OD-H, hexane/iso-propanol 99.6:0.4, flow $0.9 \mathrm{ml} / \mathrm{min}$ ): $\mathrm{t}_{1}=$ $15.66 \mathrm{~min}, \mathrm{t}_{2}=17.15 \mathrm{~min}$. 


\section{(E)-2-(Thiophen-2-yl)-1-nitro-1-propene:}

${ }^{1}$ H NMR (300 MHz, $\left.\mathbf{C D C l}_{3}, 25{ }^{\circ} \mathbf{C}\right): \delta=7.57$ (q, $\left.J=1.3 \mathrm{~Hz}, 1 \mathrm{H}, \mathrm{C} 1-H\right), 7.46-7.50(\mathrm{~m}$, 2H, arom. $H$ ), $7.13(\mathrm{dd}, J=5.0,3.7 \mathrm{~Hz}, 1 \mathrm{H}$, arom. $H), 2.70(\mathrm{~d}, J=1.3 \mathrm{~Hz}, 3 \mathrm{H}, M e)$.

${ }^{13} \mathbf{C}$ NMR (75 MHz, $\left.\mathbf{C D C l}_{3}, 25{ }^{\circ} \mathbf{C}\right): \delta=142.8,140.4,133.6,129.5,129.1,128.5,18.0$.

IR (film): $v=3108,1600,1504,1423,1378,1355,1334,1318,1251,1061,905,856$, $818,712 \mathrm{~cm}^{-1}$.

Anal. Calcd for $\mathbf{C}_{7} \mathbf{H}_{7} \mathrm{NO}_{2} \mathrm{~S}$ : C, 49.69; H, 4.17; N, 8.28 Found: C, 49.94; H, 4.37; N, 8.24.

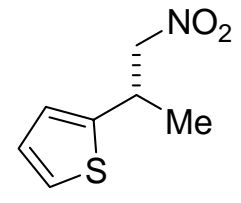

(R)-2-(Thiophen-2-yl)-1-nitro-propane:

${ }^{1} \mathbf{H}$ NMR (300 MHz, $\left.\mathbf{C D C l}_{3}, \mathbf{2 5}^{\circ} \mathbf{C}\right): \delta=7.22(\mathrm{dd}, J=5.3,1.2 \mathrm{~Hz}, 1 \mathrm{H}$, arom. $H), 6.96$ (dd, $J=5.3,3.4,1 \mathrm{H}$, arom. $H), 6.90(\mathrm{~m}, 1 \mathrm{H}$, arom. $H), 4.58(\mathrm{dd}, J=12.1,7.2 \mathrm{~Hz}, 1 \mathrm{H}$, C1- $\left.H^{1}\right), 4.48\left(\mathrm{dd}, J=12.1,8.1 \mathrm{~Hz}, 1 \mathrm{H}, \mathrm{C} 1-H^{2}\right), 3.97(\mathrm{~m}, 1 \mathrm{H}, \mathrm{C} 2-H), 1.47(\mathrm{~d}, J=6.9 \mathrm{~Hz}$, $3 \mathrm{H}, \mathrm{Me})$.

${ }^{13} \mathrm{C}$ NMR (75 MHz, $\left.\mathbf{C D C l}_{3}, 25{ }^{\circ} \mathbf{C}\right): \delta=143.8,126.9,124.4,124.2,82.1,34.2,19.8$.

IR (film): $v=2973,1552,1455,1430,1384,1240,1113,1040,850,772,702 \mathrm{~cm}^{-1}$.

Anal. Calcd for $\mathrm{C}_{7} \mathrm{H}_{9} \mathrm{NO}_{2} \mathrm{~S}: \mathrm{C}, 49.10 ; \mathrm{H}, 5.30 ; \mathrm{N}, 8.18$. Found: $\mathrm{C}, 49.28 ; \mathrm{H}, 5.37 ; \mathrm{N}$, 8.30 .

HPLC (two serial Chiracel OD-H, hexane/iso-propanol 99.6:0.4, flow $0.9 \mathrm{ml} / \mathrm{min}$ ): $\mathrm{t}_{1}=$ $29.32 \mathrm{~min}, \mathrm{t}_{2}=31.84 \mathrm{~min}$. 
<smiles>CC(C)/C(=C/NO)c1ccccc1</smiles>

\section{(Z)-3-Methyl-2-phenyl-1-nitro-1-butene:}

${ }^{1}$ H NMR (300 MHz, $\left.\mathbf{C D C l}_{3}, 25{ }^{\circ} \mathbf{C}\right): \delta=7.34-7.43(\mathrm{~m}, 3 \mathrm{H}$, arom. H), 7.07-7.14 (m, 2H, arom. $H), 7.00(\mathrm{~d}, J=1.3 \mathrm{~Hz}, 1 \mathrm{H}, \mathrm{C} 1-H), 2.72(\mathrm{~m}, 1 \mathrm{H}, \mathrm{C} 3-H), 1.13(\mathrm{~d}, J=6.9 \mathrm{~Hz}, 6 \mathrm{H}$, $\left.\mathrm{C} 3 \mathrm{Me}_{2}\right)$.

${ }^{13} \mathrm{C}$ NMR (75 $\left.\mathbf{M H z}, \mathbf{C D C l}_{3}, 25{ }^{\circ} \mathbf{C}\right): \delta=157.5,135.9,134.4,128.2,128.2,126.5,35.8$, 20.8 .

IR (film): $v=2970,2934,2876,1636,1524,1467,1444,1344,779,746,701 \mathrm{~cm}^{-1}$.

Anal. Calcd for $\mathbf{C}_{11} \mathbf{H}_{13} \mathbf{N O}_{2}: C, 69.09 ; \mathrm{H}, 6.85 ; \mathrm{N}, 7.32$ Found: C, 69.10; H, 6.65; N, 7.14 .<smiles>CC(C)C(C[N+](=O)[O-])c1ccccc1</smiles>

\section{(S)-3-Methyl-2-phenyl-1-nitro-butane:}

${ }^{1} \mathbf{H}$ NMR (300 MHz, $\left.\mathbf{C D C l}_{3}, 2{ }^{\circ} \mathbf{C}\right): \delta=7.22-7.34(\mathrm{~m}, 3 \mathrm{H}$, arom. H), 7.12-7.17 (m, 2H, arom. $H), 4.77\left(\mathrm{dd}, J=12.5,5.9 \mathrm{~Hz}, 1 \mathrm{H}, \mathrm{C} 1-H^{1}\right), 4.64(\mathrm{dd}, J=12.1,10.0 \mathrm{~Hz}, 1 \mathrm{H}, \mathrm{C} 1-$ $\left.H^{2}\right), 3.23(\mathrm{~m}, 1 \mathrm{H}, \mathrm{C} 2-H), 1.96(\mathrm{~m}, 1 \mathrm{H}, \mathrm{C} 3-H), 1.01\left(\mathrm{~d}, J=6.9 \mathrm{~Hz}, 3 \mathrm{H}, \mathrm{C} 3 M e^{1}\right), 0.81(\mathrm{~d}, J$ $\left.=6.9 \mathrm{~Hz}, 3 \mathrm{H}, \mathrm{C} 3 M e^{2}\right)$.

${ }^{13} \mathbf{C}$ NMR (75 $\left.\mathbf{M H z}, \mathbf{C D C l}_{3}, 25{ }^{\circ} \mathbf{C}\right): \delta=138.5,128.5,128.0,127.3,79.1,51.1,31.4$, 20.7, 20.3.

IR (film): $v=3032,2965,1556,1496,1455,1435,1381,755,702 \mathrm{~cm}^{-1}$. Anal. Calcd for $\mathbf{C}_{11} \mathbf{H}_{15} \mathbf{N O}_{2}$ : C, 68.37; H, 7.82; N, 7.25. Found: C, 68.40; H, 7.72; N, 7.23 
HPLC (Chiracel OD-H, hexane/iso-propanol 98:2, flow $0.9 \mathrm{ml} / \mathrm{min}$ ): $\mathrm{t}_{1}=6.70 \mathrm{~min}, \mathrm{t}_{2}=$ $9.86 \mathrm{~min}$.

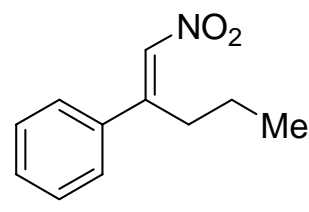

(E)-2-Phenyl-1-nitro-1-pentene:

${ }^{1} \mathbf{H}$ NMR (300 MHz, $\left.\mathbf{C D C l}_{3}, 25{ }^{\circ} \mathbf{C}\right): \delta=7.43(\mathrm{~m}, 5 \mathrm{H}$, arom. $H), 7.20(\mathrm{~m}, 1 \mathrm{H}, \mathrm{C} 1-H)$, 3.01 (m, 2H, C3- $H_{2}$ ), 1.53 (m, 2H, C4-H), 0.97 (t, $J=7.5 \mathrm{~Hz}, 3 \mathrm{H}, M e$ ).

${ }^{13} \mathbf{C}$ NMR (75 MHz, $\left.\mathbf{C D C l}_{3}, \mathbf{2 5}{ }^{\circ} \mathbf{C}\right): \delta=154.2,137.2,136.0,130.0,128.9,127.0,33.1$, $21.9,14.2$.

IR (film): $v=2964,2934,2874,1614,1575,1518,1444,1339,934,838,770,744,697$ $\mathrm{cm}^{-1}$.

Anal. Calcd for $\mathrm{C}_{11} \mathbf{H}_{13} \mathrm{NO}_{2}: \mathrm{C}, 69.09 ; \mathrm{H}, 6.85 ; \mathrm{N}, 7.32$. Found: $\mathrm{C}, 68.82 ; \mathrm{H}, 6.84 ; \mathrm{N}$, 7.17 .

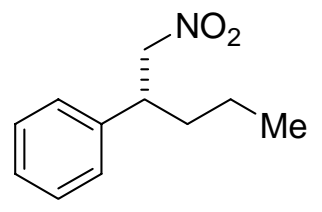

(R)-2-Phenyl-1-nitro-pentane:

${ }^{1} \mathbf{H}$ NMR (300 MHz, $\left.\mathbf{C D C l}_{3}, \mathbf{2 5}^{\circ} \mathbf{C}\right): \delta=7.23-7.37(\mathrm{~m}, 3 \mathrm{H}$, arom. H), 7.17-7.22 (m, 2H, arom. $H$ ), $4.58\left(\mathrm{dd}, J=12.1,7.8 \mathrm{~Hz}, 1 \mathrm{H}, \mathrm{C} 1-H^{1}\right), 4.53\left(\mathrm{dd}, J=12.1,8.1 \mathrm{~Hz}, 1 \mathrm{H}, \mathrm{C} 1-H^{2}\right)$, $3.47(\mathrm{~m}, 2 \mathrm{H}, \mathrm{C} 2-\mathrm{H}), 1.67\left(\mathrm{~m}, 2 \mathrm{H}, \mathrm{C} 3-\mathrm{H}_{2}\right), 1.22\left(\mathrm{~m}, 2 \mathrm{H}, \mathrm{C} 4-\mathrm{H}_{2}\right), 0.88$ (t, J = 7.2 Hz, 3H, $\mathrm{Me})$.

${ }^{13} \mathbf{C}$ NMR (75 MHz, $\left.\mathbf{C D C l}_{3}, 25{ }^{\circ} \mathbf{C}\right): \delta=139.4,128.8,127.4,127.4,81.0,44.2,35.2$, 20.2, 13.9. 
IR (film): $v=3032,2961,2934,2874,1553,1496,1455,1431,1380,763,701 \mathrm{~cm}^{-1}$.

Anal. Calcd for $\mathbf{C}_{11} \mathbf{H}_{15} \mathbf{N O}_{2}: \mathrm{C}, 68.37 ; \mathrm{H}, 7.82 ; \mathrm{N}, 7.25$. Found: C, 68.22; H, 7.77; N, 7.21 .

HPLC (Chiracel OD-H, hexane/iso-propanol 98:2, flow $0.9 \mathrm{ml} / \mathrm{min}$ ): $\mathrm{t}_{1}=6.66 \mathrm{~min}, \mathrm{t}_{2}=$ $9.23 \mathrm{~min}$.<smiles>O=[N+]([O-])C=C(CO)c1ccccc1</smiles>

(Z)-3-Nitro-2-phenylprop-2-en-1-ol:

${ }^{1}$ H NMR (300 MHz, $\left.\mathbf{C D C l}_{3}, 25{ }^{\circ} \mathbf{C}\right): \delta=7.40-7.55(\mathrm{~m}, 5 \mathrm{H}$, arom. $H), 7.21(\mathrm{~m}, 1 \mathrm{H}$, $\mathrm{CHNO}_{2}$ ), 4.87 (m, 2H, $\left.\mathrm{CH}_{2} \mathrm{OH}\right), 3.02$ (br s, $1 \mathrm{H}, \mathrm{OH}$ ).

${ }^{13} \mathrm{C}$ NMR (75 MHz, $\left.\mathbf{C D C l}_{3}, 25{ }^{\circ} \mathrm{C}\right): \delta=151.9,136.8,134.9,130.4,128.9,127.3,60.2$.

IR (film): $v=3428,3101,3062,1704,1615,1556,1519,1445,1339,1263,1054,1032$, $977,920,843,769,739,698 \mathrm{~cm}^{-1}$.

HRMS (ESI, KOAc) m/e: Calcd for $\left(\mathrm{M}+\mathrm{K}^{+}\right) \mathrm{C}_{9} \mathrm{H}_{9} \mathrm{NO}_{3} \mathrm{~K}$ : 218.02140. Found: 218.02105 .<smiles>O=[N+]([O-])CC(CO)c1ccccc1</smiles>

(S)-3-Nitro-2-phenylpropan-1-ol:

${ }^{1} \mathbf{H}$ NMR (300 MHz, $\left.\mathbf{C D C l}_{3}, 2{ }^{\circ} \mathbf{C}\right): \delta=7.30-7.40(\mathrm{~m}, 3 \mathrm{H}$, arom. H), 7.22-7.26 (m, 2H, arom. $H$ ), $4.89\left(\mathrm{dd}, J=12.8,6.8 \mathrm{~Hz}, 1 \mathrm{H}, \mathrm{CH}^{1} \mathrm{NO}_{2}\right), 4.70(\mathrm{dd}, J=12.8,7.8 \mathrm{~Hz}, 1 \mathrm{H}$ $\left.\mathrm{CH}^{1} \mathrm{NO}_{2}\right), 3.80-3.97\left(\mathrm{~m}, 2 \mathrm{H}, \mathrm{CH}_{2} \mathrm{OH}\right), 3.71(\mathrm{~m}, 1 \mathrm{H}, \mathrm{C}(2) H), 1.68(\mathrm{t}, J=5.3 \mathrm{~Hz}, 1 \mathrm{H}$, $\mathrm{OH})$. 
${ }^{13} \mathbf{C}$ NMR (75 MHz, $\left.\mathbf{C D C l}_{3}, 25{ }^{\circ} \mathbf{C}\right): \delta=136.9,129.1,128.1,127.7,77.1,64.3,46.3$.

IR (film): $v=3397,3032,2941,2884,1604,1551,1496,1455,1433,1380,1198,1062$, $1029,762,701 \mathrm{~cm}^{-1}$.

HRMS (ESI, KOAc) m/e: Calcd for $\left(\mathrm{M}+\mathrm{K}^{+}\right) \mathrm{C}_{9} \mathrm{H}_{11} \mathrm{NO}_{3} \mathrm{~K}: 220.03705$ Found: 220.03709 .

Anal. Calcd for $\mathbf{C}_{9} \mathbf{H}_{11} \mathbf{N O}_{3}$ : C, 59.66, H, 6.12, N, 7.73. Found: C, 59.85, H, 6.24, N, 7.53.

HPLC (OAc derivative, two serial Chiracel OD-H, hexane/iso-propanol 90:10, flow 0.9 $\mathrm{ml} / \mathrm{min}): \mathrm{t}_{1}=31.52 \mathrm{~min}, \mathrm{t}_{2}=34.51 \mathrm{~min}$. 


\section{HPLC Traces}<smiles>CC(C)C(C[N+](=O)[O-])c1ccccc1</smiles>
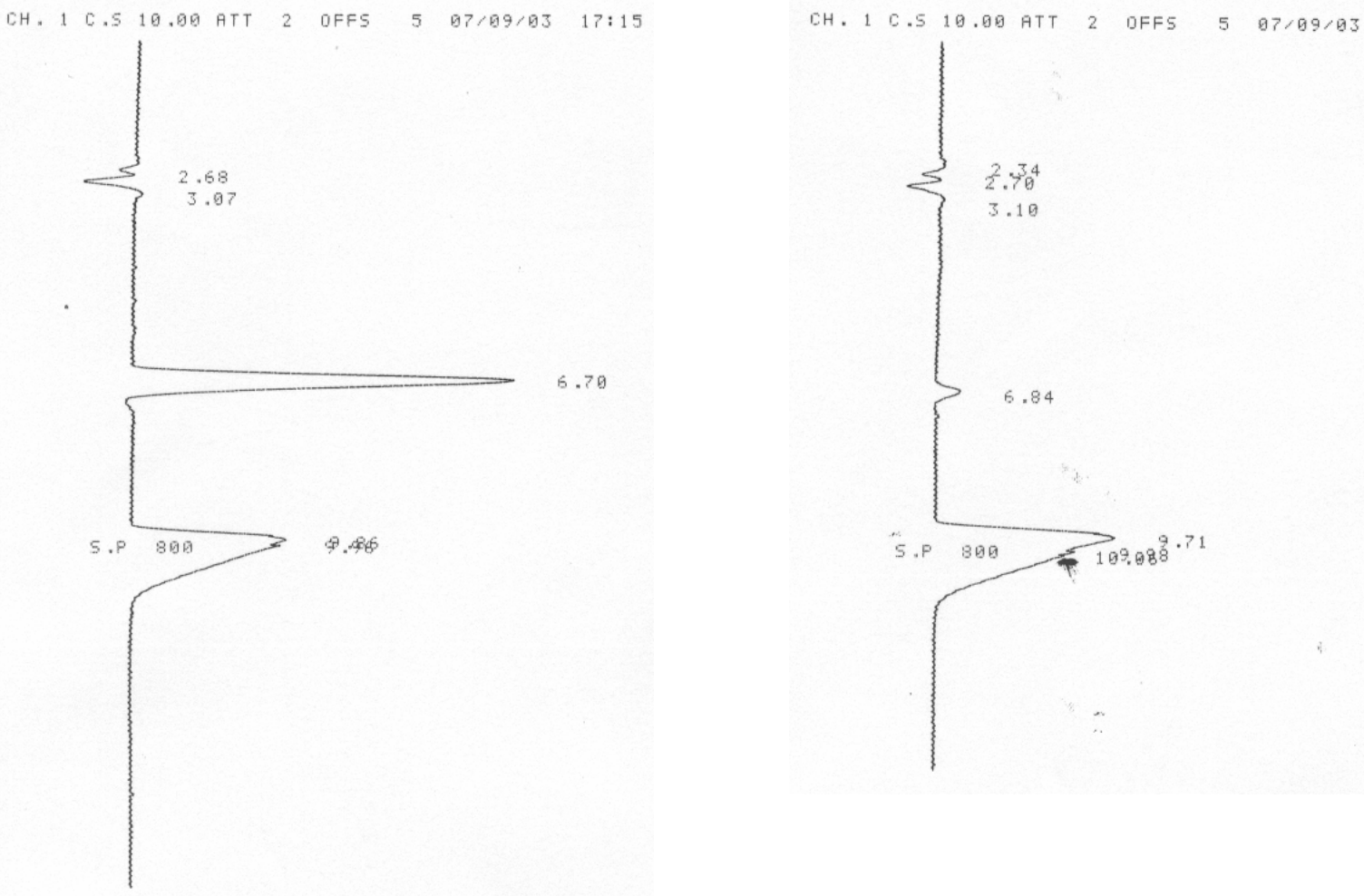

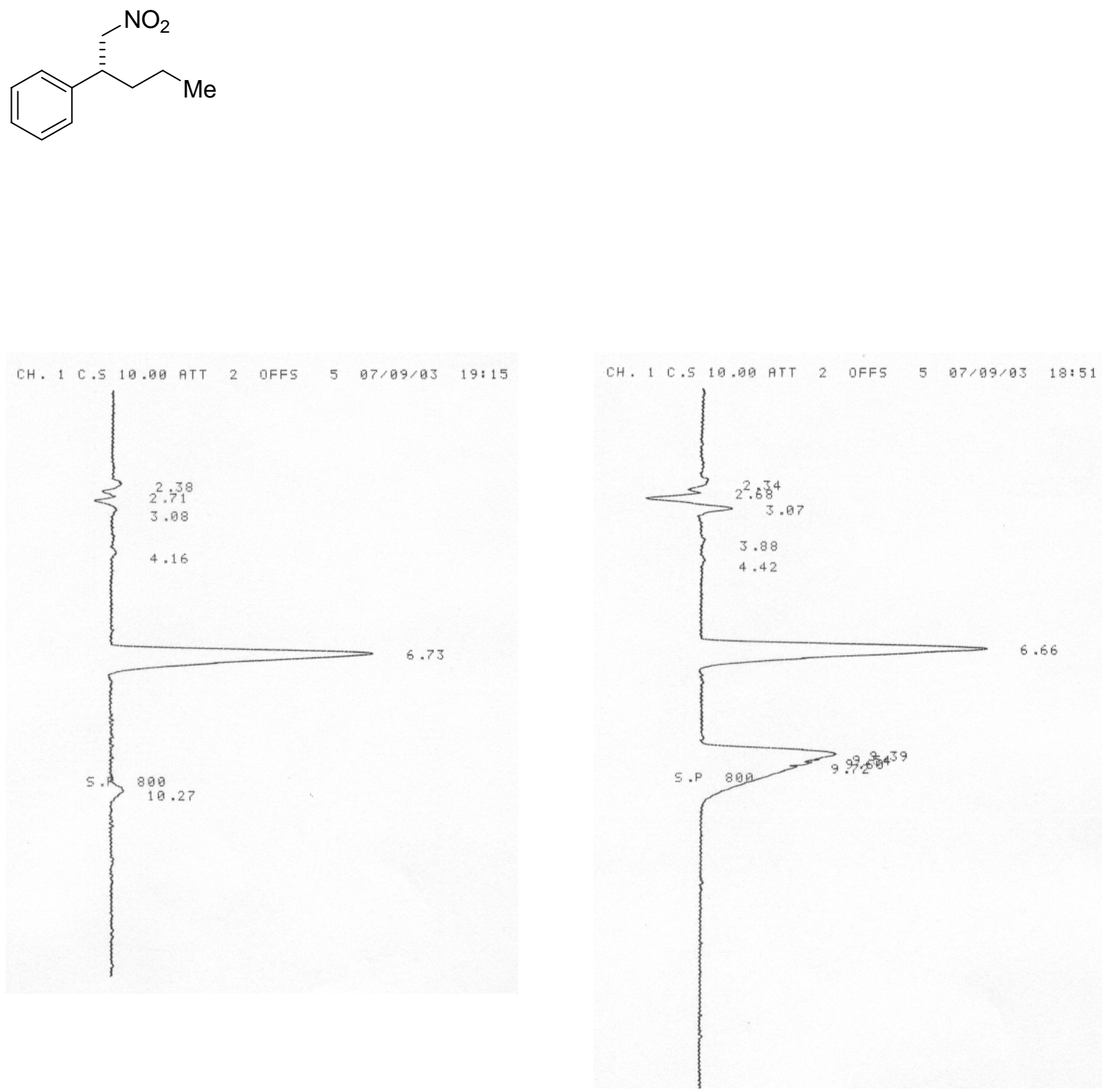

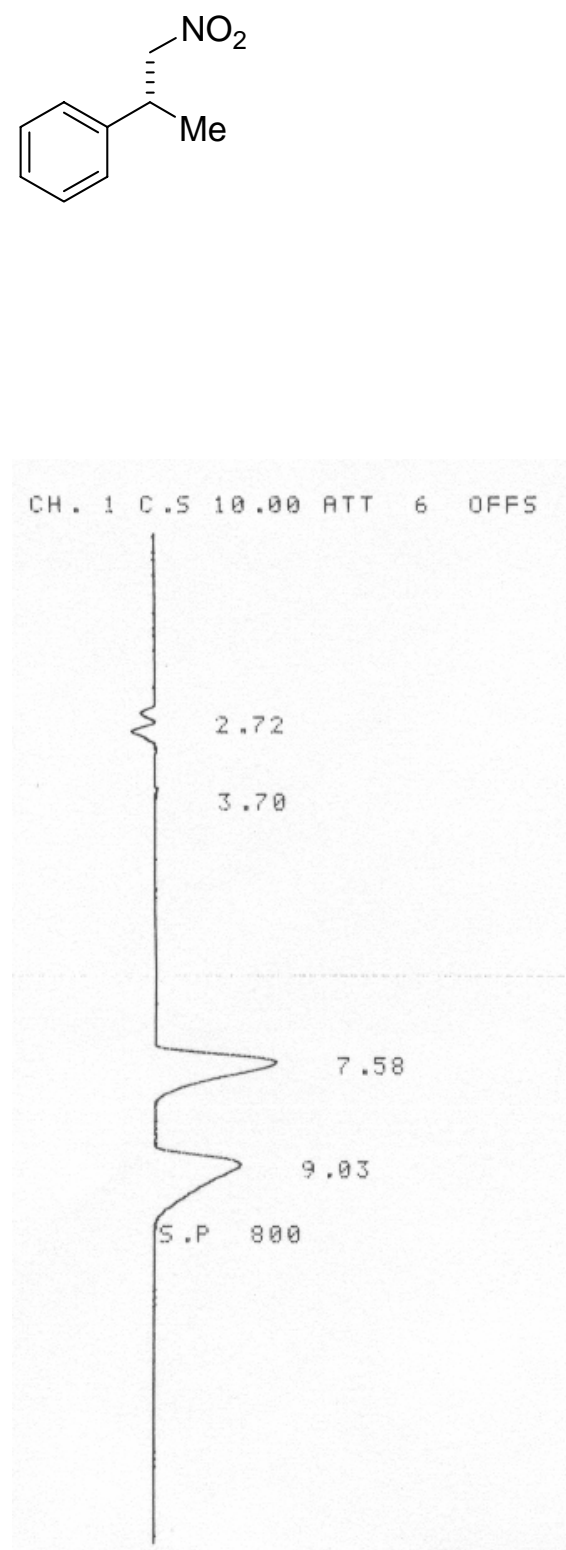

CH. 1 C.S 1 日. . A ATT 6 OFFS

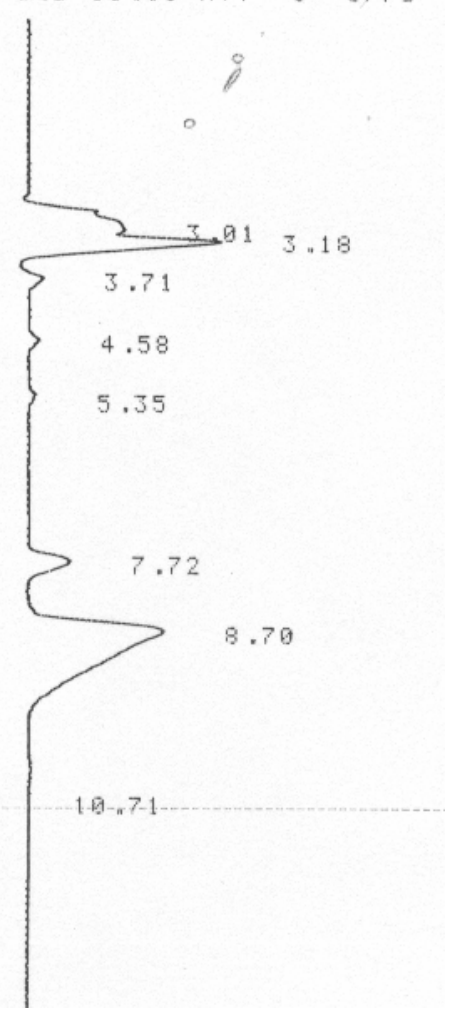



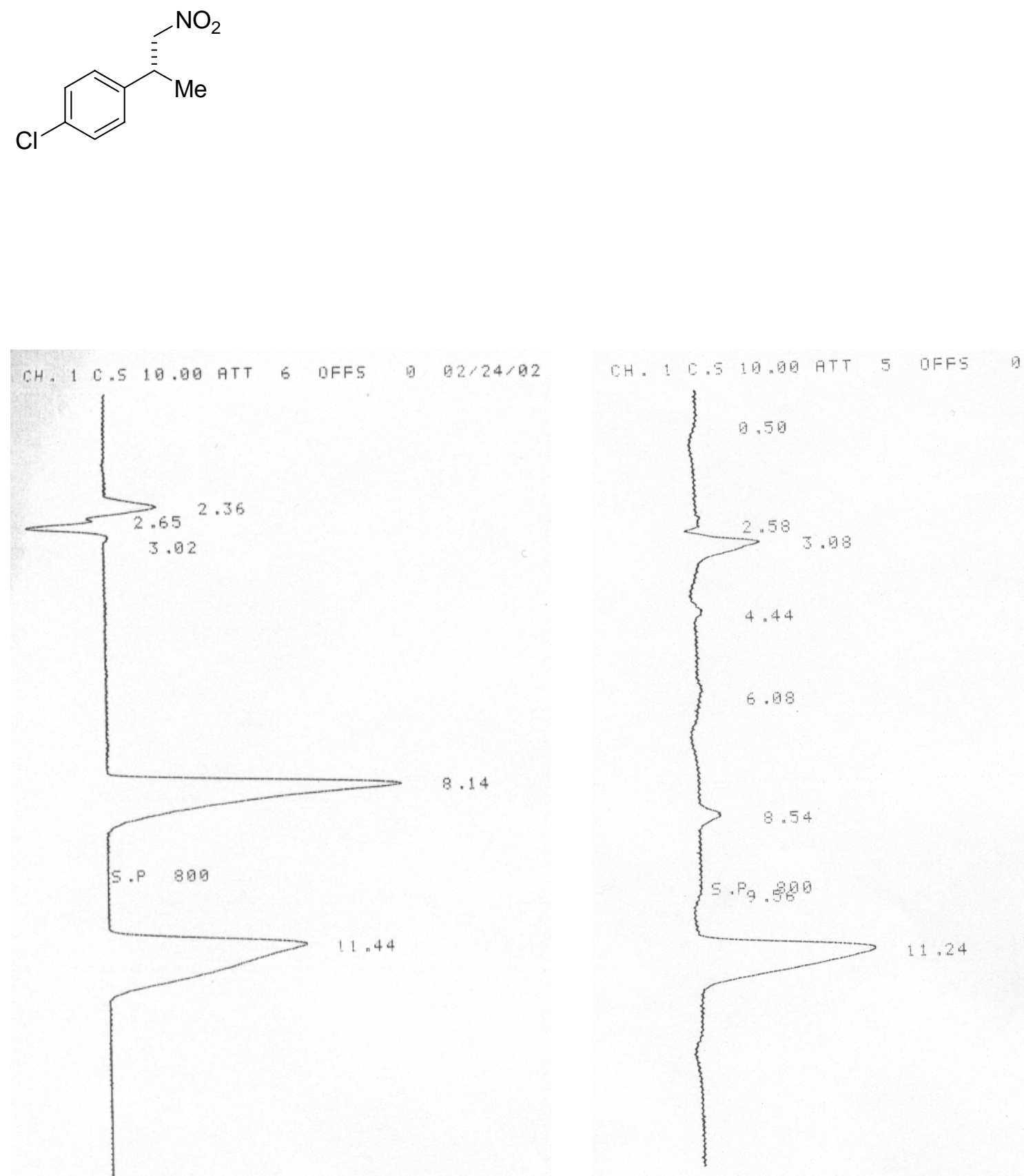

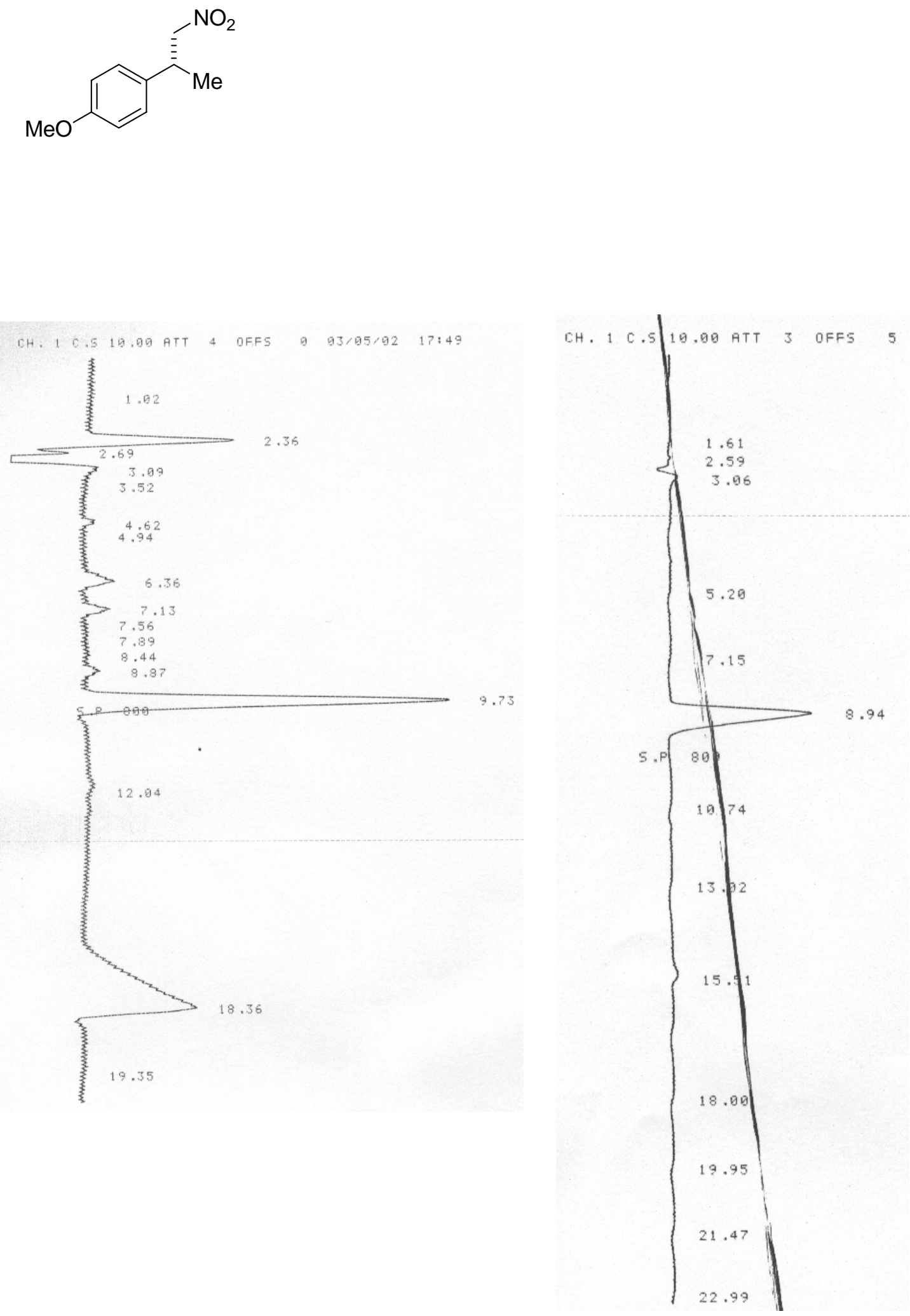

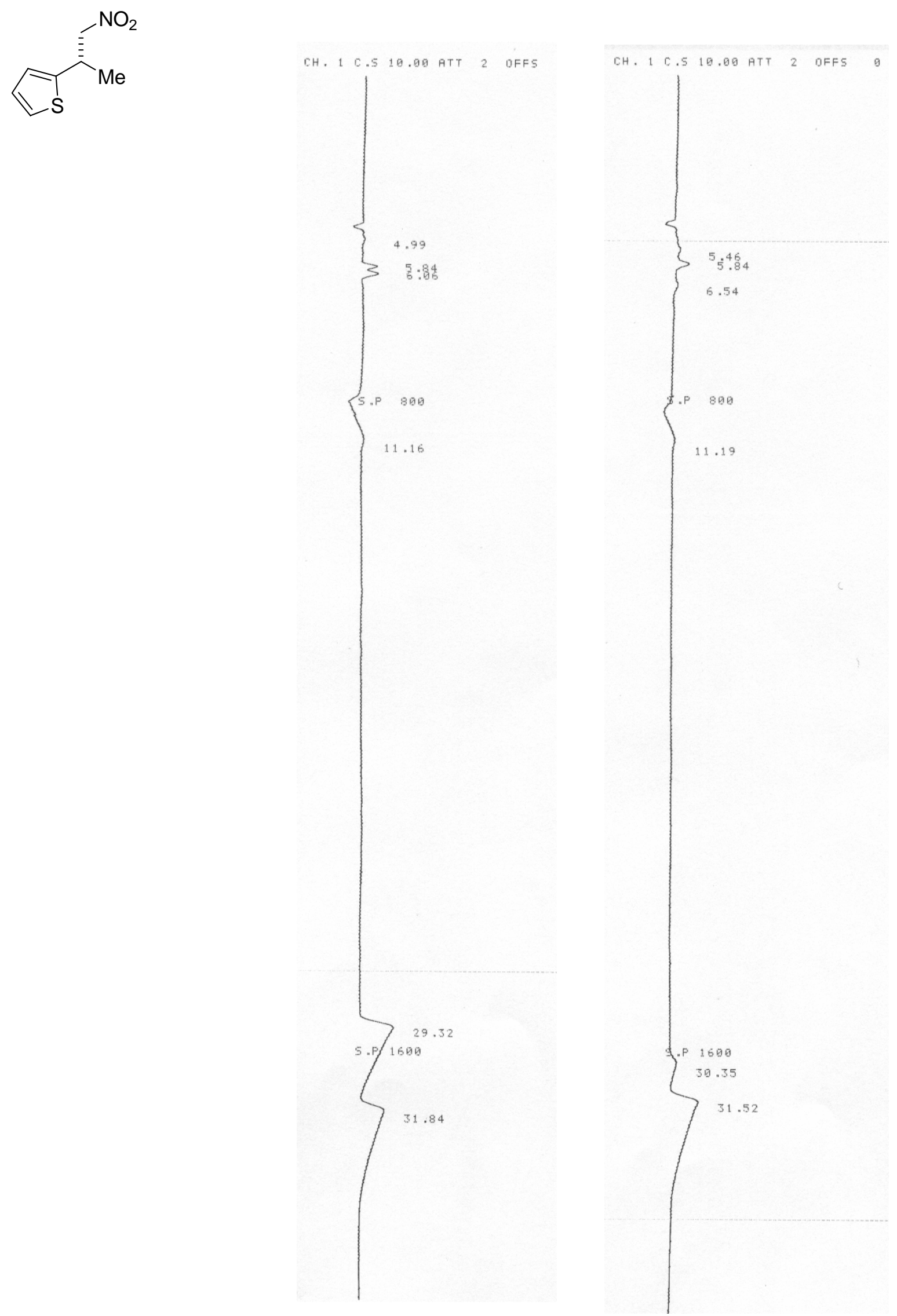

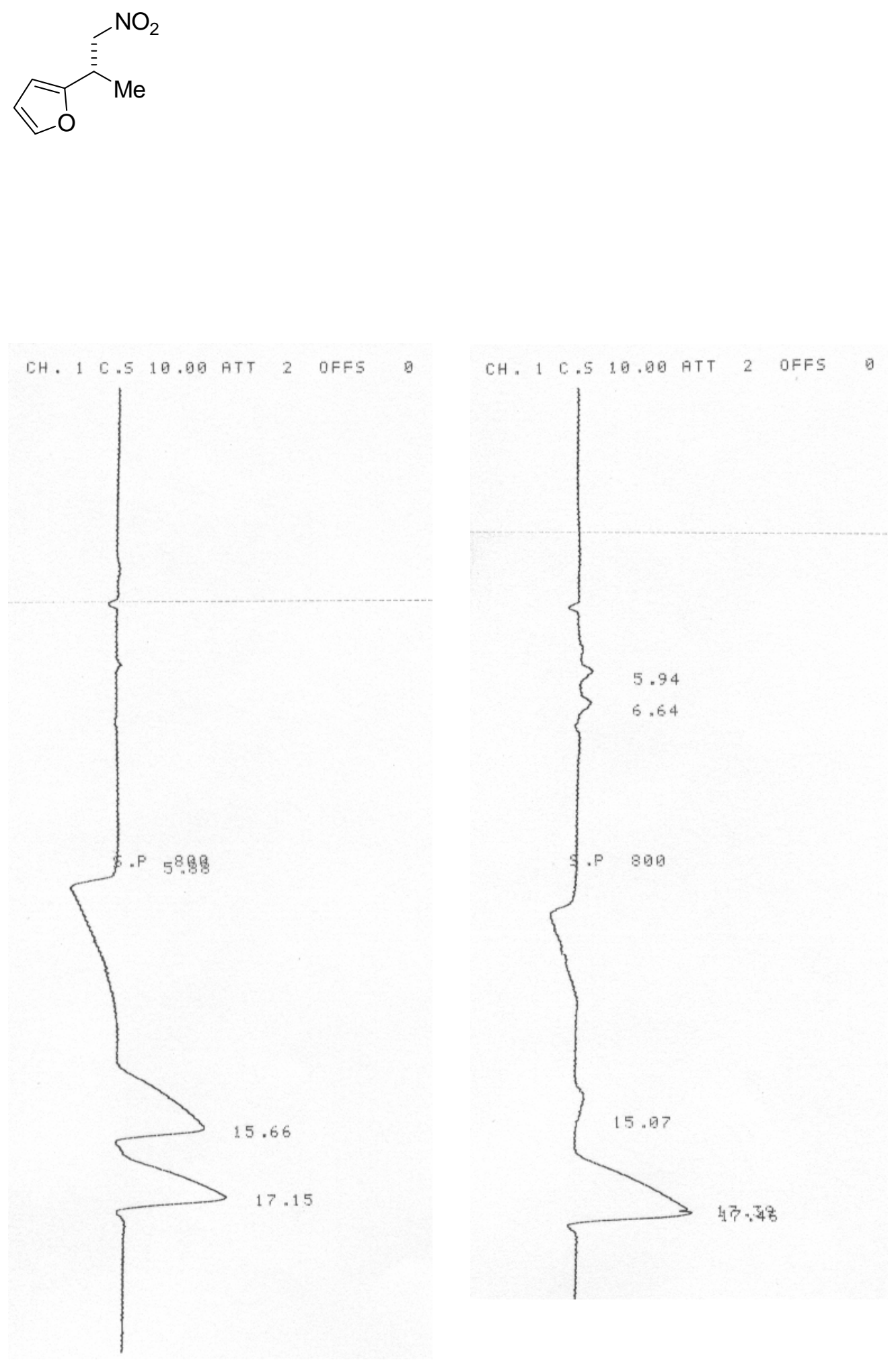

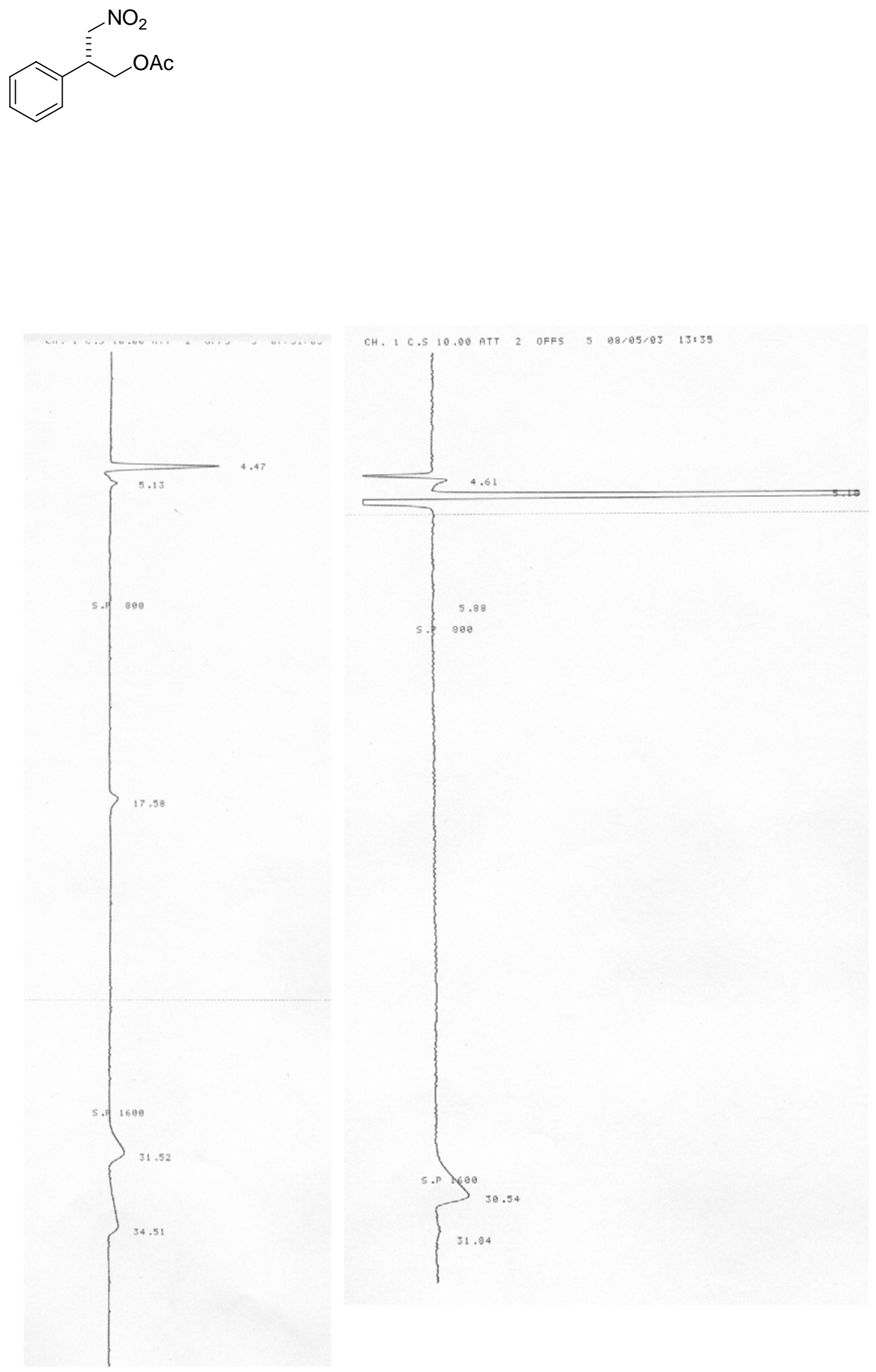